(2) Open Access Full Text Article

ORIGINAL RESEARCH

\title{
Comparative Study of the Effects of Atypical Antipsychotic Drugs on Plasma and Urine Biomarkers of Oxidative Stress in Schizophrenic Patients
}

This article was published in the following Dove Press journal:

Neuropsychiatric Disease and Treatment

\author{
Anna Dietrich-Muszalska (D) \\ Joanna Kolodziejczyk- \\ Czepas $^{2}$ \\ Pawel Nowak ${ }^{2}$ \\ 'Medical University of Lodz, Department \\ of Biological Psychiatry and \\ Neurophysiology, Lodz, Poland; \\ ${ }^{2}$ University of Lodz, Department of \\ General Biochemistry, Lodz, Poland
}

Purpose: Evidence that antipsychotic drugs (ADs) can affect oxidative stress estimated with various biomarkers in schizophrenic patients is controversial and limited. Therefore, in the present study, we assessed the ability of six atypical ADs (clozapine, olanzapine, quetiapine, risperidone, aripiprazole, and ziprasidone) used in schizophrenia treatment to modulate oxidative damage to different biomolecules such as lipids and proteins.

Patients and Methods: We measured the levels of oxidative stress markers in plasma and urine: total antioxidant capacity by FRAP (according to a modified method of Benzie \& Strain), thiobarbituric acid reactive species - TBARS (spectrophotometric method), 4-hydroxy-2-nonenal (4-HNE) (OxiSelect ${ }^{\mathrm{TM}}$ HNE Adduct Competitive ELISA Kit), 3-nitrotyrosine (3-NT) (OxiSelect ${ }^{\mathrm{TM}}$ Nitrotyrosine ELISA Kit) in plasma, and F2-isoprostanes $\left(\right.$ BIOXYTECH $^{\circledR}$ Urinary 8-epi-Prostaglandin F2 $\alpha$ ) in the urine of 60 schizophrenic patients (before and after treatment) and in 30 healthy subjects.

Results: Our results showed that in schizophrenic patients levels of lipid peroxidation markers (TBARS, F2-isoprostanes) were higher than in healthy subjects but FRAP in schizophrenic patients was lower than in healthy controls and increased after 4-week treatment with tested ADs. A 4-week treatment with ADs caused the improvement of psychopathology symptoms estimated by Positive and Negative Syndrome Scale (PANSS) that was accompanied by decreased lipid peroxidation (F2-isoprostanes, TBARS; $\mathrm{p}=2.9 \times 10^{-6}, \mathrm{p}=7.6 \times 10^{-5}$, respectively) and an increase in total antioxidative capacity (FRAP) $\left(\mathrm{p}=5.16 \times 10^{-16}\right)$.

Conclusion: Atypical antipsychotics especially clozapine, olanzapine and quetiapine demonstrate the effective outcome of antipsychotic treatment, beneficial antioxidative action by reducing lipid peroxidation and increased total plasma antioxidant activity.

Keywords: schizophrenia, antipsychotics, F2-isoprostanes, TAC, other oxidative markers

\section{Introduction}

Schizophrenia is a multifactorial psychiatric illness with diverse clinical manifestations characterized by cognitive, emotional and behavioral disturbances affecting about $1 \%$ of the population worldwide. The manifestations include, among others, positive symptoms, negative symptoms and working memory and other cognitive deficits. ${ }^{1,2}$ The etiopathogenesis of schizophrenia has not yet been explained, and a multitude of theories have been proposed over the years. Schizophrenia is a neurodevelopmental disease; there is evidence supporting a progressive and
Correspondence: Anna Dietrich-Muszalska Department of Biological Psychiatry and Neurophysiology Medical University of Lodz, Lodz, 92-215, Poland, Mazowiecka 6/8

Tel +691881787

Fax +48422725652

Email tzn_lodz@post.pl
Neuropsychiatric Disease and Treatment 2021:17 555-565

DovePress $f y$ in $\boldsymbol{t}$

http://doi.org/10.2147/NDT.S283395 
possibly neurodegenerative process. ${ }^{3}$ Clinical observations complemented by neuroimaging and genetic studies prove the neurodevelopmental hypothesis. ${ }^{4}$ On the other hand, neuropathological studies support the neurodegenerative hypothesis. A hypothesis, called the progressive neurodevelopmental model, has also emerged. ${ }^{5}$ Cell dysfunction, apoptotic process, decreased neurogenesis and neuroplasticity are described in the brain of schizophrenic individuals. ${ }^{6-8}$ There has been accumulating evidence supporting the involvement of oxidative/nitrative stress due to increased activity of reactive oxygen species (ROS) and reactive nitrogen species (RNS) as well as decreased antioxidant protection in the development and course of the disease; leading to damage to lipids, proteins, and DNA. ${ }^{9}$ Antipsychotic medications are standard care for patients and ADs are commonly used in the treatment of schizophrenia and other psychiatric disorders. Progressive pathophysiological processes occurring within the brain of patients with schizophrenia may be modulated by ADs that have the potential for neuroprotection or modulation of chemical neurotransmission. Efficacy of ADs, their mechanism of action and potential to exert effects on cellular processes in the brain may play a neuroprotective role in schizophrenia. ${ }^{10}$ It is known that atypical antipsychotics block dopamine D2 receptors as well as serotonin receptors (eg 5HT2A) and due to these processes they perform their main therapeutic function. ${ }^{10}$ Their impact on neuronal signaling systems is complex. ADs can be divided into the first generation; typical ADs (TADs, eg haloperidol) and the second generation; atypical ADs (AADs, eg olanzapine, clozapine, quetiapine, aripiprazole, risperidone and ziprasidone). Although TADs improve positive symptoms, they are marginally effective for negative symptoms and ineffective for cognitive deficits. ${ }^{11}$ TADs (eg haloperidol) treat some of the symptoms of schizophrenia, such as delusions and hallucinations, but can have some side effects including extrapyramidal signs, tardive dyskinesia and hyperprolactinemia. AADs block both dopamine and serotonin binding to their specific receptors. ${ }^{12}$ Some of them may have additional therapeutic properties such as cognitive enhancement, reduction of negative symptoms, prevention of enhanced relapse of disease, regression and clinical deterioration. ${ }^{13}$ AADs are increasingly prescribed, often replacing typical TADs. ${ }^{14}$ Recent evidence suggests that AADs may mitigate or reverse some of the morphological changes observed in schizophrenic patients such as gray matter volume reduction, caudate hypertrophy, white matter volume increase and decrease in dendritic spine density in the prefrontal cortex. ${ }^{10}$ However, treatment with some AADs causes side effects associated with body weight gain and development of metabolic syndrome, agranulocytosis, and hepatotoxicity. ${ }^{15}$ Although the biological mechanisms involved in the side effects induced by antipsychotics remain to be elucidated, oxidative stress may be involved in these mechanisms. There are numerous clinical and experimental reports demonstrating changes of oxidant/ antioxidant balance in response to the treatment with ADs. In rats, not only haloperidol but also other AADs (clozapine, olanzapine, risperidone) induced a significant decrease in the activity of superoxide dismutase (SOD) and catalase (CAT), and increased lipid peroxidation. ${ }^{16}$ The reduced activities of antioxidant enzymes observed in schizophrenic patients and free radical-mediated changes in the structure of different biomolecules measured by the increased level of specific oxidative stress markers may contribute to specific aspects of schizophrenia symptomatology and partly to the complications caused by the treatment with antipsychotics. To date, a majority of studies have presented an alteration in the antioxidant defense system (eg activities of enzymes) and in the level of lipid peroxidation in vitro as well as in animal model; fewer studies showed peroxidation/nitration products derived from cellular biomolecules such as lipids or proteins in schizophrenic patients treated with antipsychotics. ${ }^{9,17-20}$ It cannot be excluded that atypical antipsychotics such as clozapine, quetiapine, olanzapine, risperidone, aripiprazole, and ziprasidone may differentially affect the oxidative stress markers since they do not work in the same way. Therefore, the purpose of our study was to evaluate the effect of 4-week treatment with antipsychotics on the total antioxidant capacity of plasma (ferric reducing ability of plasma - FRAP) and the levels of peroxidation products of lipids in urine and plasma (F2-isoprostanes, TBARS, 4-HNE, and 3-nitrotyrosine) in schizophrenic patients.

\section{Materials and Methods Subjects}

The study involved 30 healthy controls and 60 patients with schizophrenia who met the eligibility criteria (inclusion/exclusion criteria) shown in Table 1. The inclusion and exclusion criteria for healthy controls were the same as for schizophrenic patients (excluding AADs treatment). There was no significant difference between patients and control group on any characteristics such as sex, age, body mass index and smoking. 
Table I Inclusion and Exclusion Criteria of Schizophrenic Patients

Inclusion Criteria
22 years < age > 38 years ( 19 females and 4 I males); similar in race/ethnicity (Caucasian)
Recruitment: schizophrenics hospitalized in the 2 nd Psychiatric Department of Medical University in Lodz, Poland
DSM-IV diagnosis of schizophrenia (acute phase)
Provision of written informed consent
Balanced diet (meat and vegetables)
Body mass index (BMI) in normal range (I8.5-24.9)
Antipsychotic monotherapy with study AADs
No additional drugs especially no antidepressants or mood stabilizers
Exclusion Criteria
Neurological or physical disorders; especially neurodegenerative disorders, addictions, history of head injuries, infections, allergies, and lipid or
carbohydrate metabolism disorders
Movement disorders (eg extrapyramidal symptoms and tardive dyskinesia)
Additional drugs, including antibiotics and other anti-inflammatory drugs within 2 weeks prior to the study
Electroconvulsive therapy within the previous 6 months
Narcotics use
Alcohol use within I week before the blood collection
Tobacco smoking within at least 10 days before urine sample collection (controlled by cotinine test)
Any supplementation with antioxidants (plants and pharmaceuticals) and vitamins during the study
Any supplementation with polyunsaturated fatty acids within 6 months before the study
Pregnant and breast-feeding women

\section{Methods}

Patients with a diagnosis of schizophrenia (paranoid type) according to DSM-IV criteria (American Psychiatric Association, 1994 ${ }^{21}$ were interviewed with a special questionnaire (treatment, course of diseases) and clinically assessed for movement disorders, including dyskinesia and other extrapyramidal symptoms (using the SimpsonAngus Scale (SAS) and the Barnes Akathisia Rating Scale (BARS)). ${ }^{22,23}$ The patients with schizophrenia were assessed according to the Positive and Negative Syndrome Scale (PANSS; Kay et al, 1987). ${ }^{24}$

Healthy controls were screened assessing their medical history and using medical structured interviews; psychiatric examination using the M.I.N.I.-Mini International Neuropsychiatric Interview ${ }^{25}$ was performed. Both study groups had neurological and physical examinations and laboratory tests, including biomarkers of oxidative stress, glucose and total cholesterol, LDL, HDL, triglycerides concentrations, performed.

All participants granted written informed consent before beginning of the study after receiving a full explanation of this study. The study was approved by the Committee for Research on Human Subjects of the Medical University of Lodz (RNN/899/2000) and conducted according to the ethical obligations of the Declaration of Helsinki.
The detailed characteristics of studied groups are shown in Table 2.

\section{Study Protocol \\ Patients}

The study had an open-label design with run-in period and consisted of daily patients observation and 4 visits as below:

At visit 1 (preliminary): consent to participation in the study was obtained, medical interviews and assessments in terms of inclusion criteria were conducted.

Table 2 Clinical Characteristics of Patients with Schizophrenia and Healthy Controls

\begin{tabular}{|l|l|}
\hline $\begin{array}{l}\text { Patients with schizophrenia }(\mathrm{n}=60) \text {; treatment } \\
\text { with (stable dose for acute episode) }{ }^{26} \text { clozapine } \\
\text { or olanzapine or quetiapine or aripiprazole or } \\
\text { risperidone or ziprasidone ( } \mathrm{n}=10 \text { for each drug) }\end{array}$ & $\begin{array}{l}\text { Control subject } \\
(\mathrm{n}=30)\end{array}$ \\
$\begin{array}{l}\text { The clinical response was defined as } \geq 20 \% \\
\text { reduction in PANSS positive symptoms score. }\end{array}$ & \\
\hline Sex, M/F $4 \mathrm{I} / 19$ & $18 / 12$ \\
Age (years), average \pm SD: $30.4 \pm 3.2$ & $30.0 \pm 3.1$ \\
Duration of illness (years) $8.4 \pm 4.3$ & NA \\
Smokers/non-smokers $8 / 52$ & $0 / 30$ \\
\hline
\end{tabular}

Abbreviation: NA, not applicable. 
At visit 2 (1st day): the patients had physical examination and neuropsychiatric assessment including psychometric scales (eg PANSS); the beginning of the run-in period.

At visit 3 (baseline; after run-in period and after 2 weeks of drug application, when the final stable dose of AADs for acute episode of schizophrenia was reached): neuropsychiatric and physical assessments, including psychometric scale (eg PANSS), were conducted; blood and urine samples were collected and laboratory tests, including biomarkers of oxidative stress, were performed. The patients were under no influence of emergency drugs eg anxiolytics, hypnotics, analgesics, etc.

At visit 4 (endpoint; after 4-week treatment with stable doses for acute episode of schizophrenia): physical examination and neuropsychiatric assessment, including psychometric scale (eg PANSS) were conducted; blood and urine samples were collected and laboratory tests, including biomarkers, were performed. The patients were under no influence of emergency drugs eg anxiolytics, hypnotics, analgesics, etc.

\section{Healthy Controls}

At visit 1 (preliminary) consent to participation in the study was obtained, medical interviews and assessments in terms of inclusion criteria were conducted.

At visit 2 (1st day) the psychiatric examination using the M.I.N.I-Mini International Neuropsychiatric Interview, neurological and physical examinations were performed; blood and urine samples were collected and laboratory tests, including biomarkers, were performed.

\section{Isolation of Plasma and Collection of Urine Samples}

Blood samples $(2 \times 7.5 \mathrm{~mL})$ from healthy controls and patients were collected into the solution of ACD (citric $\mathrm{acid} /$ citrate/dextrose; $5: 1 \mathrm{v} / \mathrm{v}$ ) between 8.00 and $8.30 \mathrm{a} . \mathrm{m}$. and centrifuged for $20 \mathrm{~min}$ at $2500 \mathrm{rpm}$ and $20^{\circ} \mathrm{C}$ in the Sigma $3 \mathrm{~K} 30$ centrifuge to obtain plasma for the further determination of TBARS, 4-HNE, FRAP and 3-NT. F2isoprostanes were determined in urine samples. To that end, about $10 \mathrm{~mL}$ of spot morning urine was collected according to the procedure by Roberts and Morrow (2000). ${ }^{28,29}$ Collected samples were stored at $-80^{\circ} \mathrm{C}$ for no longer than 6 months. Each donor's sample was assayed in duplicate.

\section{Experimental Procedure}

Evaluation of Lipid Peroxidation

Determination of Urinary Isoprostanes

Measurements were carried out using an enzyme immunoassay for urinary isoprostane, ie OxisResearch ${ }^{\mathrm{TM}}$ kit $\left(\right.$ BIOXYTECH $^{\circledR}$ Urinary 8-epi-Prostaglandin F2 $\alpha$, OXIS Health Products, Inc., Foster City, USA), according to the protocol of the manufacturer. The method is based on a competitive enzyme-linked immunoassay (ELISA), detecting the 8-epi-prostaglandin- $\mathrm{F}_{2 \alpha}$. The quantitative analysis of the obtained results was done with the use of a standard curve (ranging from 0.05 to $100 \mathrm{ng} / \mathrm{mL}$ of 8-epi-prostaglandin- $\mathrm{F}_{2 \alpha}$ ).

Measurements of the Thiobarbituric Acid-Reactive Substances (TBARS)

Samples of plasma were transferred to an equal volume of $20 \%(\mathrm{v} / \mathrm{v})$ cold trichloroacetic acid in $0.6 \mathrm{M} \mathrm{HCl}$ and centrifuged at $1200 \times \mathrm{g}$ for $15 \mathrm{~min}$. One volume of clear supernatant was mixed with 0.2 volume of $0.12 \mathrm{M}$ thiobarbituric acid in $0.26 \mathrm{M}$ Tris, $\mathrm{pH} 7.0$, and immersed in a boiling water bath for $15 \mathrm{~min}$. The absorbance was measured in the SEMCO spectrophotometer (at $535 \mathrm{~nm}$ in 1-cm cuvettes), according to the modification of the Rice-Evans method. ${ }^{30,31}$ The TBARS concentrations expressed in $\mu \mathrm{mol} / \mathrm{L}$ were calculated based on the absorbance value, using the molar extinction coefficient for TBARS $\left(\varepsilon=1.56 \times 10^{5} \mathrm{M}^{-1} \times \mathrm{cm}^{-1}\right)$.

\section{Determination of Hydroxynonenal (4-HNE) in Plasma}

The assay was carried out using OxiSelect ${ }^{\mathrm{TM}}$ HNE Adduct Competitive ELISA Kit (Cell Biolabs Inc., San Diego, USA), according to the protocol of the manufacturer. The method is based on an immunodetection of the protein-HNE adducts. Concentrations of the adducts in the examined samples were quantified using the predetermined HNEBSA standard curve (ranging from 1.56 to $200 \mu \mathrm{g} / \mathrm{mL}$ ).

\section{Determination of 3-Nitrotyrosine in Plasma}

Determination of plasma 3-nitrotyrosine was carried out with the use of OxiSelect ${ }^{\mathrm{TM}}$ Nitrotyrosine ELISA Kit (Cell Biolabs Inc., San Diego, USA). The procedure was based on a competitive enzyme-linked immunosorbent assay. The procedure was executed according to the protocol of the manufacturer. Quantitative determination of 3-nitrotyrosine in the examined samples was performed on the basis of a standard curve, prepared with increasing concentration $(0.049$ to $200 \mu \mathrm{g} / \mathrm{mL})$ of nitrated bovine serum albumin, which corresponded to 3-nitrotyrosine concentrations of $1.95-8000 \mathrm{nM}$. 


\section{Evaluation of Total Antioxidant Capacity of Plasma}

The total antioxidant capacity (TAC) of blood plasma was evaluated with FRAP (ferric reducing ability of plasma) method dependent on non-enzymatic antioxidants and determined according to a modified method by Benzie \& Strain, 1996. ${ }^{32}$ The procedure was standardized at the Department of General Biochemistry, University of Lodz. ${ }^{33}$

\section{Statistical Analysis}

All the values in this study were expressed as the mean \pm standard error of the mean. In order to eliminate outliers, Grubbs' test was performed. All data had normal distribution as evaluated with the Shapiro-Wilk test. The data (F2isoprostanes, TBARS, 4-HNE level, 3-NT and FRAP) from healthy subjects and patients with schizophrenia were analyzed with Student's $t$-test. The significance of differences for study marker levels (baseline and endpoint at 4 weeks after therapy with stable doses) was calculated using the paired Student's $t$-test. The data at ADs were analyzed with multifactorial variance analysis (two-way analysis of variance [ANOVA] test). Post hoc comparisons for the oxidative markers levels were carried out with Fisher's Least Significant Difference test (LSD test) STATISTICA v.10 by StatSoft (Palo Alto, CA, USA) was used.

\section{Results}

Our results show that levels of two of the studied oxidative markers in schizophrenic patients (F2 - isoprostanes and TBARS) were higher than in healthy controls (Figure 1A and B), whereas total antioxidant capacity
A

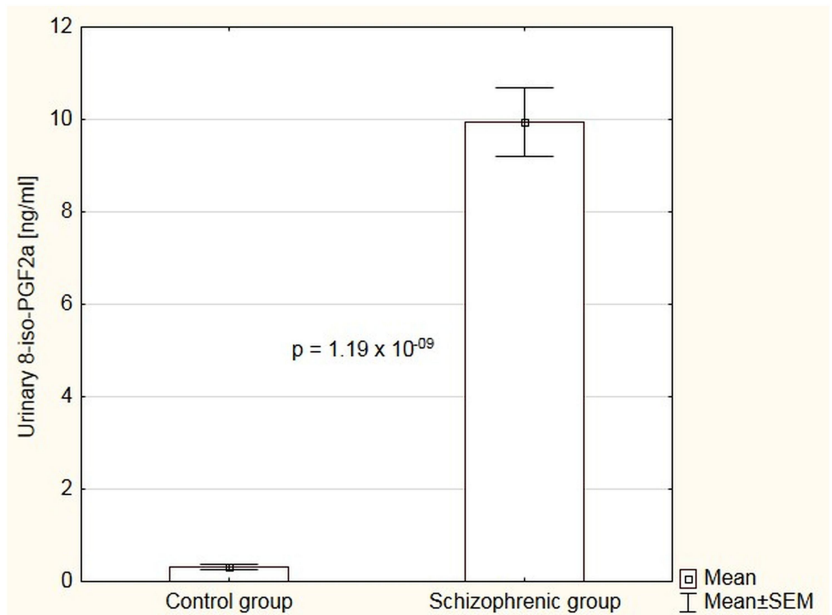

B

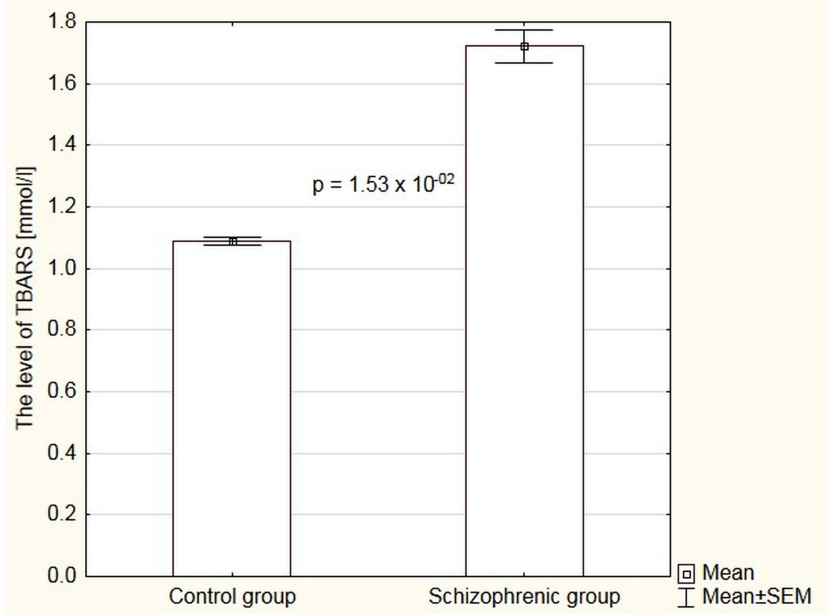

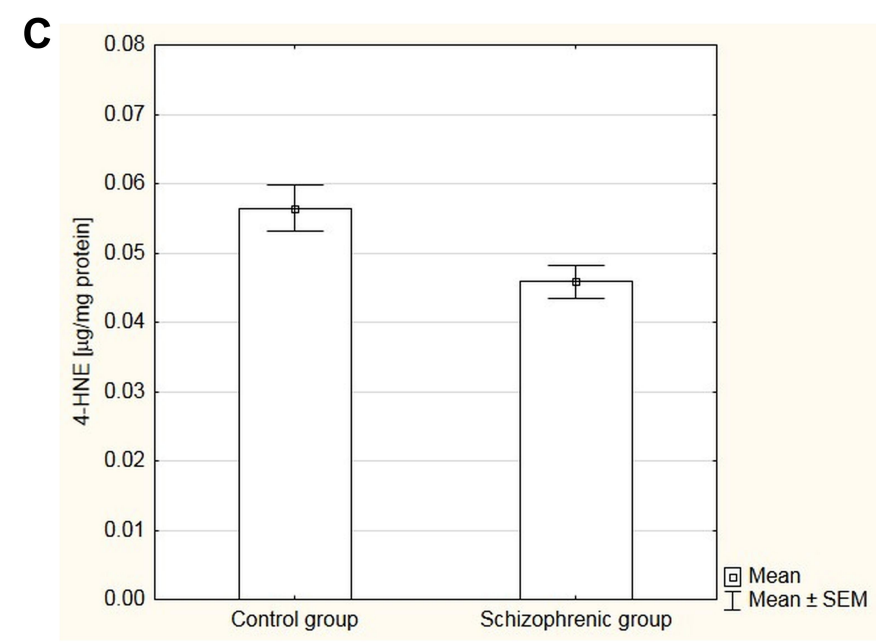

Figure I The comparison of lipid peroxidation markers between healthy controls $(n=30)$ and schizophrenic patients ( $n=60)$ in plasma and urine. The level of lipid peroxidation markers in urine and plasma of controls and schizophrenic patients (mean \pm SEM) in all calculations Student's $t$-test was used: F2-isoprostanes level in urine 0.33 \pm 0.06 vs.9.94 $\pm 0.74 ; p=1.19 \times 10^{-09}(\mathbf{A})$, TBARS $1.09 \pm 0.09$ vs $1.72 \pm 0.08 ; p=1.53 \times 10^{-02}$ (B), 4-HNE $p \leq 0.05$ (C). 
of plasma estimated by FRAP assay in schizophrenic patients was lower (Figure 2) than in the controls. 4-HNE levels in both groups did not differ significantly (Figure 1C). Similarly, the received results indicated that levels of 3-NT were low. All the obtained values were (0.1-1.5 $\mu \mathrm{g} / \mathrm{mg}$ protein) about the limit of detection, with no statistical differences between the control and schizophrenic groups. The effect of treatment with selected AADs on the level of markers of oxidative stress (F2 isoprostanes, TBARS and FRAP) are presented in
Figure 3. After 4-week treatment (with stable doses) of schizophrenic patients with selected antipsychotics, a reduction in levels of $\mathrm{F} 2$-isoprostanes (Figure 3A) and TBARS (Figure 3B) was observed $\left(\mathrm{p}=2.9 \times 10^{-6}\right.$, $\mathrm{p}=7.6 \times 10^{-5}$, respectively), while the levels of $4-\mathrm{HNE}$ and 3-NT were not changed. An increase in FRAP was noted after 4-week treatment in patients compared to baseline $\left(\mathrm{p}=5.16 \times 10^{-16}\right)$ (Figure $\left.3 \mathrm{C}\right)$. The ANOVA test (two-way analysis of variance test) showed that the effects of atypical antipsychotics (clozapine, olanzapine,

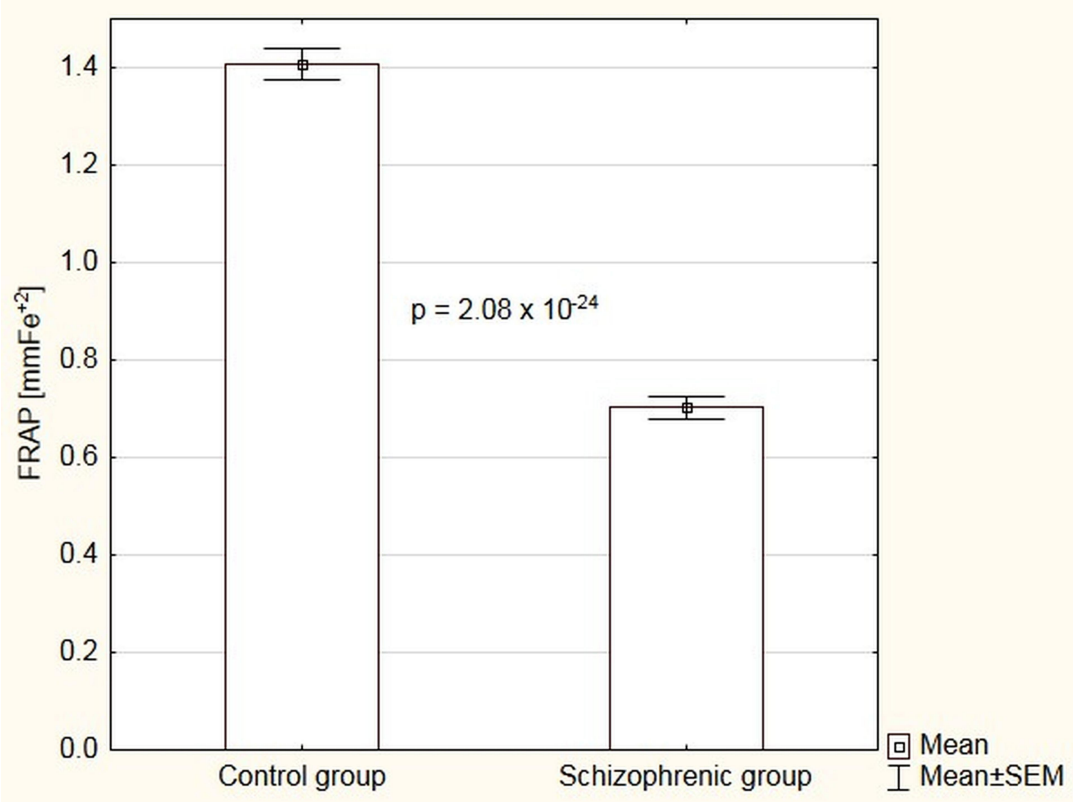

Figure 2 The comparison of FRAP level between control group $(n=30)$ and schizophrenic patients $(n=60)$. (Mean \pm SEM) $I .4 I \pm 0.03$ vs $0.67 \pm 0.02 ; p=2.08 \times 10^{-24}($ Student $s t-$ test).

A

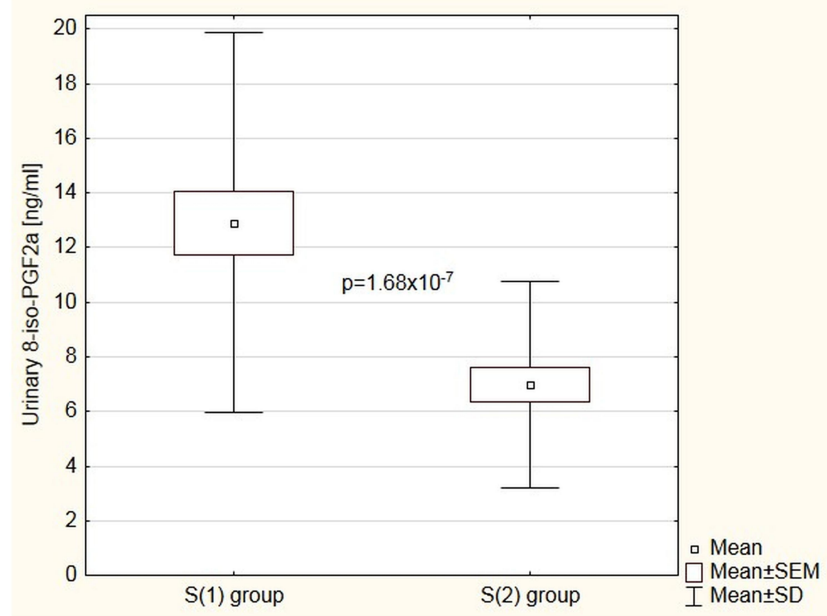

B

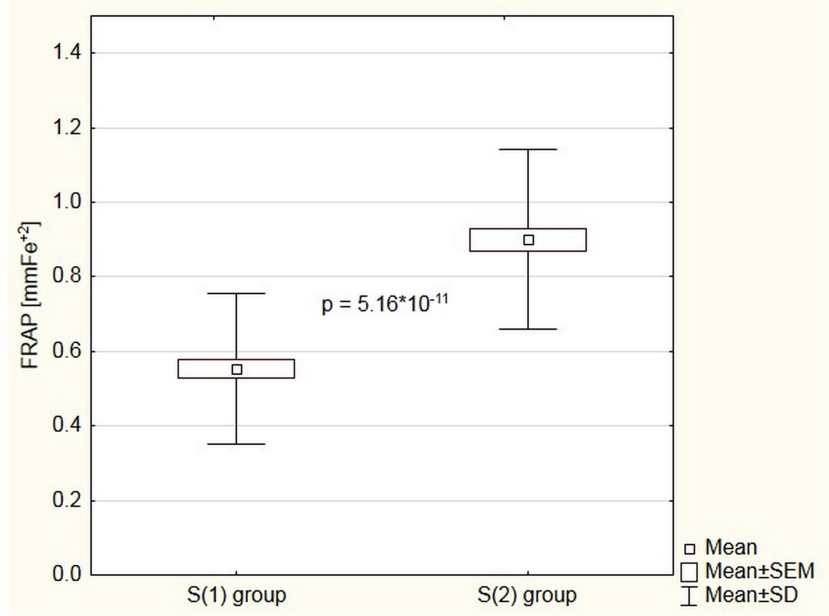

Figure 3 The effect of AADs on the level of F2-isoprostanes in urine (A) and FRAP (B) in plasma of schizophrenic patients at baseline S(I) and after 4 weeks of treatment (endpoint) S(2). The significance of differences for study marker levels (baseline and endpoint at 4 weeks after therapy with stable doses of AADs) was calculated using the paired Student's $t$-test; for F2-isoprostanes level $p=1.68 \times 10^{-07}(\mathbf{A})$, for FRAP $p=5.16 \times 10^{-11}$ (B). 
quetiapine, risperidone, aripiprazole, and ziprasidone) on oxidative stress markers were significantly different for F2-isoprostanes and FRAP $\left(\mathrm{p}=0.003 ; \mathrm{p}=4.53 \times 10^{-8}\right.$, respectively). The post hoc analysis (LSD test) showed that clozapine, olanzapine and quetiapine exhibited the best antioxidant properties: the highest increase was in FRAP and decrease in F2-isoprostanes at endpoint compared to baseline $\left(\mathrm{p}=1.0 \times 10^{-5} ; \mathrm{p}=0.003\right.$, respectively). The effect of treatment with clozapine, olanzapine, and quetiapine on the level of oxidative stress markers (F2isoprostanes and FRAP) is presented in Figure 4A and B.

A

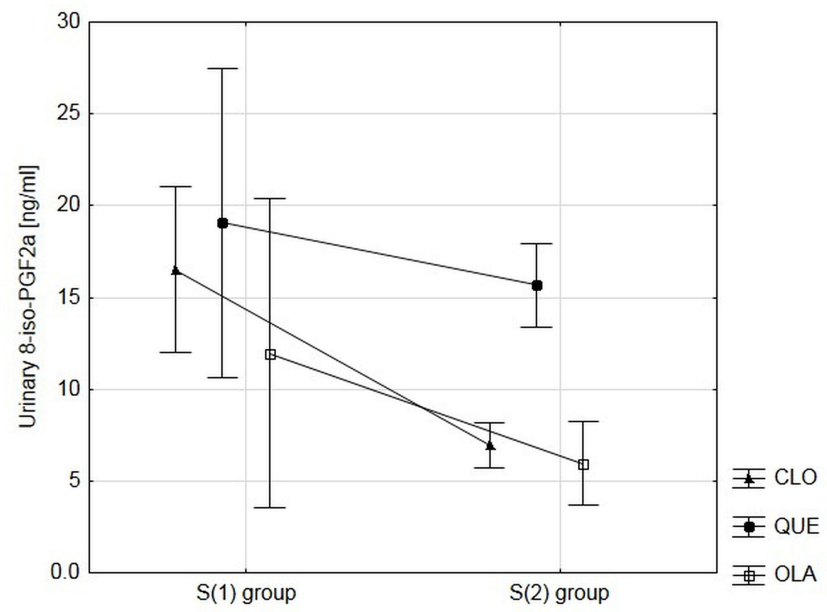

Effects of 4-week treatment with stable doses of clozapine, olanzapine and quetiapine were connected with changes of biomarkers: decrease in F2-isoprostanes (differences between baseline (S1) and endpoint (S2)) were significant about $42 \%, 50 \%, 82 \%$, respectively, and increase in FRAP (differences between baseline (S1) and endpoint (S2)) were significant about 72\%, 65\%, $81 \%$, respectively (Figure 5). Weaker antioxidant effects were exhibited by aripiprazole, risperidone and ziprasidone (differences between baseline (S1) and endpoint (S2) were not significant).

B

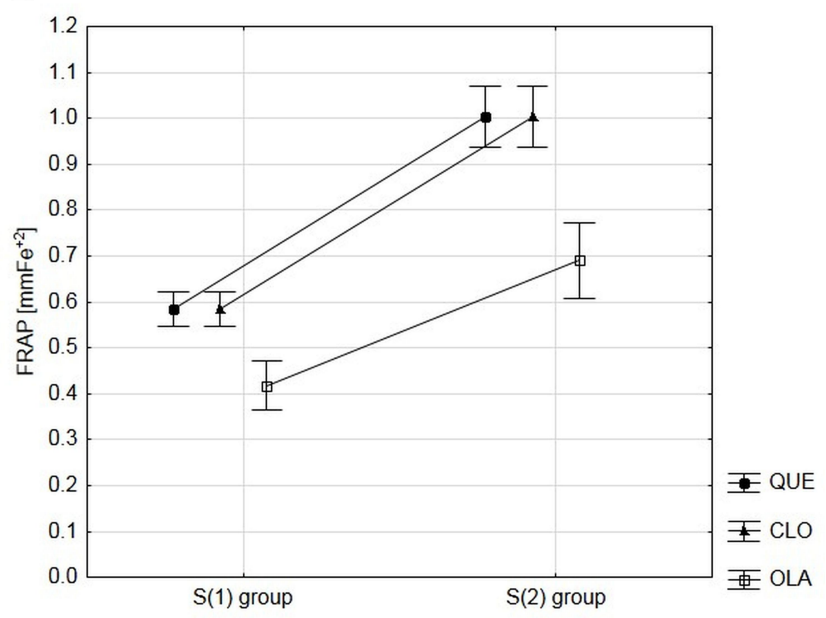

Figure 4 The effect of clozapine (CLO), olanzapine (OLA), and quetiapine (QUE) on the level of F2-isoprostanes in urine (A) and FRAP in plasma (B) of schizophrenic patients at baseline $\mathrm{S}(\mathrm{I})$ and after 4-week treatment (endpoint) $\mathrm{S}(2)$. (A) The effect of CLO, OLA, and QUE on the level of F2-isoprostanes in urine of schizophrenic patients at baseline $S(I)$ and endpoint $S(2)$; mean \pm SEM: for CLO 16.52+4.5; 6.95+0.1 .2; $p=0,0005$; for QUE $p<0.05$; for OLA $p<0.05$. (B) The effect of CLO, OLA, and QUE on the level of FRAP in plasma of schizophrenic patients at baseline $S(I)$ and endpoint $S(2)$; mean \pm SEM: for CLO $0.58 \pm 0.04 ; 1.01+0.07, p=1.1 \times 10^{-8}$; for $Q U E 0.47 \pm 0.06$; $0.84 \pm$ $0.06, p=0.0002$; for OLA $0.42 \pm 0.05 ; 0.69 \pm 0.08, p=0.005$.

A

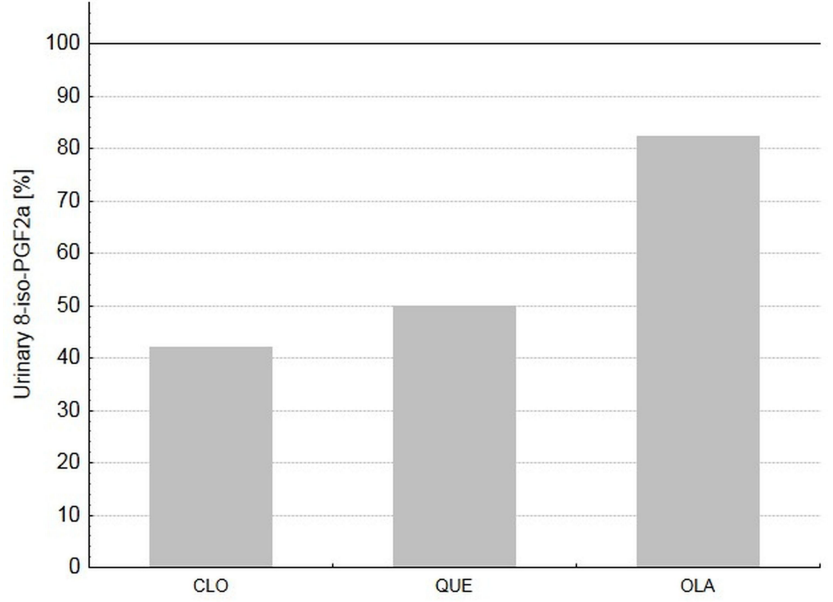

B

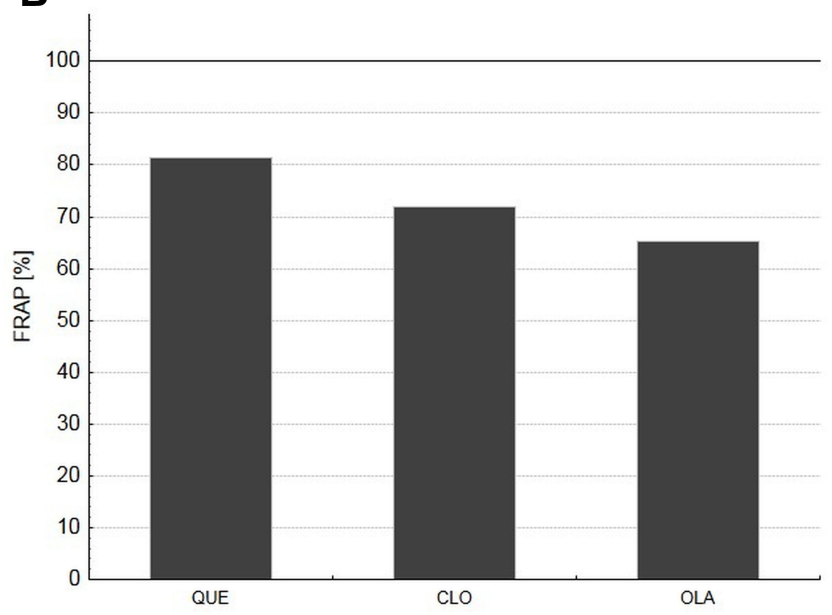

Figure 5 An increase of FRAP level (A) and decrease of F2-isoprostanes level (B) in schizophrenic patients at baseline $\mathrm{S}(\mathrm{I})$ and after 4-week treatment (endpoint) S(2) expressed as a percentage. 


\section{Discussion}

Treatment with antipsychotic drugs leads to considerable alteration of oxidation-reduction balance and may have some effects on oxidative stress in schizophrenic patients. It should be underlined that pharmacological mechanisms of several AADs action may be different. Atypical antipsychotic drugs are a class of agents that have become the most widely used to treat schizophrenia and a variety of psychoses because of their superiority regarding extrapyramidal symptoms. ${ }^{34}$ They remain the standard care for patients with schizophrenia and other psychiatric disorders despite their side effects such as metabolic syndrome, agranulocytosis or occurring less frequently than for typical antipsychotics, extrapyramidal symptoms. ${ }^{35}$ The adverse reactions associated with extrapyramidal symptoms and tardive dyskinesia (TD) have been attributed to neuronal cell damage caused by reactive oxygen species (ROS) induced partly by antipsychotics. ${ }^{36}$ The biological mechanisms underlying these side effects remain unclear. It has been hypothesized that oxidative stress induced by antipsychotics treatment may be involved in these adverse effects. Oxidative stress with the production of free radicals and oxidative damage to biomolecules may contribute to specific aspects of schizophrenic symptomatology and complications of its treatment (prominent negative symptoms, TD, parkinsonian symptoms). ${ }^{35}$ Chronic treatment with ADs may lead to an increase in free radical production, decrease in the activity of antioxidant enzymes and augmentation of damage to lipids, proteins, and nucleic acids. Regarding atypical antipsychotics, there has been increased concern about the serious metabolic side effects induced by these drugs. Clozapine has been associated with disturbances of antioxidant enzymes and oxidation of protein involved in energy metabolism and metabolic side effects. ${ }^{37}$ Bošković et al ${ }^{38}$ claimed that ADs induce oxidative stress via dopamine metabolism. This process leads to the formation of ROS that react with cellular components such as lipids, proteins, and nucleic acids. In our study, we measured the levels of biomarkers of oxidative/nitrative changes in proteins, and lipids in schizophrenic patients treated with different atypical antipsychotics. Lipid peroxidation is a major consequence of oxidative damage to a variety of cells in vivo. In our previous study, we showed that F2-isoprostanes in the urine of schizophrenic patients were increased. ${ }^{39}$ The present study showed that the level of isoprostanes was higher in the urine of schizophrenic patients than in healthy controls and it decreased after 4-week treatment with stable doses of tested AADs. TBARS or the end products of lipid peroxidation such as malondialdehyde (MDA) are widely used markers of oxidative stress in clinical studies. ${ }^{19,40-43}$ We used TBARS estimation to establish changes in lipid peroxidation in plasma of schizophrenic patients and showed that TBARS level was higher in schizophrenics than in healthy subjects but after the treatment ( 4 weeks) it significantly decreased in schizophrenics compared to baseline. $4 \mathrm{HNE}$ is a common lipid peroxidation product and a signal of oxidative stress at the cell membrane. Lipid peroxidation-derived 4-HNE easily forms covalent adducts with nucleophilic functional groups in macromolecules such as proteins, DNA, and lipids. ${ }^{44}$ Medina-Hernández et al $2007^{44}$ found higher concentrations of MDA and 4-HNE in the plasma. In our study, we did not observe a significantly increased level of 4-HNE in plasma of schizophrenic patients in comparison with healthy controls. Our results showed that the total antioxidant capacity of plasma (FRAP) in schizophrenic patients was lower than in healthy subjects but after 4 weeks of treatment with selected tested AADs (with stable doses) the level of FRAP increased and was higher than in healthy subjects and schizophrenics before the treatment (baseline). Antipsychotic drugs may affect biochemical processes in the brain, particularly the dopamine system with free radical production and oxidative and nitrative damage to brain structure. Neurons are particularly vulnerable to the damage by free radicals, ROS and RNS. Nitric oxide (NO) produced in brain mainly enzymatically by NOS (nitric oxide synthase) is involved in brain functioning and may play a dual role, both neuroprotective and neurotoxic. ${ }^{45}$ Its reaction with superoxide radical and formation of peroxynitrite leads to damaging effects. Peroxynitrite reacts with different biological molecules, damaging proteins, nucleic acids, initiating lipid peroxidation in membranes of the cells. Peroxynitrite causes mainly nitration of tyrosine residue in proteins to form 3-nitrotyrosine (3-NT) - marker of nitrative stress. ${ }^{45}$ The level of NO metabolites was found to be significantly higher in platelets of patients treated with AADs than in platelets of control subjects. ${ }^{46}$ Increased protein nitration, as measured by the level of 3-NT, was observed in the prefrontal cortex of schizophrenic patients. ${ }^{47}$ Increased protein nitration in CNS may be reflected by the changes of the level of 3-NT in blood platelets. ${ }^{46}$ In our study, the level of $3-\mathrm{NT}$ in plasma proteins of schizophrenic patients was very low; 
therefore, we did not observe any changes induced by AADs. All studied AADs approved by the Food and Drug Administration (FDA) for the treatment of schizophrenia (olanzapine, clozapine, quetiapine, aripiprazole, risperidone and ziprasidone) showed varied antioxidative activities. These findings are based on studies of antipsychotic drugs at therapeutic doses used to the treatment of an acute episode of schizophrenia. In our present study, we showed that schizophrenic patients treated with some AADs monotherapy had increased TAC level. It confirms the suggestions of other authors. ${ }^{48}$ Glica et al (2014) have shown that in chronic schizophrenic patients after clozapine or risperidone treatment the changes in plasma total antioxidant activity were observed. The changes correlated with the activities of antioxidant enzyme paraoxonase $(\mathrm{PON}){ }^{49}$ We were the first to study patients with effective improvement in AADs monotherapy. After 4-week treatment with the studied AADs drugs, we found in schizophrenics patients with improved clinical state significantly decreased F2 isoprostanes level and increased TAC level. Clozapine, olanzapine and quetiapine exhibited the best antioxidant properties. Similarly, in our in vitro studies, we demonstrated antioxidative activity of some AADs, the highest of olanzapine, ${ }^{50}$ followed by clozapine and in some cases quetiapine. ${ }^{50,51}$ In turn, in another study in plasma in vitro model, we found antioxidative properties of quetiapine. ${ }^{51}$ The other authors (Ustundag et al, 2006; Virit et al, 2009) ${ }^{48,52}$ observed both in the first episode and chronic schizophrenics that positive symptoms of schizophrenia have also been related to low antioxidative capacity. In turn Al-Chalabi et al, $2009^{53}$ described increased TAS levels in patients treated with olanzapine. Our present results indicate that treatment of schizophrenic patients with AADs leads to patients' improvement (measured by PANSS) simultaneously with the reduction of oxidative stress measured by specific biomarker levels. It should be underlined that the effects of tested drugs on the levels of oxidative stress biomarkers and the mechanisms of their action are still unclear. Therefore, further clinical and biochemical research should be carried out. This pilot study will be continued. The main problem of our paper concerns the exclusion of some patients in the study caused by the drug changes or by treatment of patients with additional antipsychotics due to the lack of improvement or insufficient effects of treatment. Taken together, our results suggest that atypical antipsychotic drugs may have a protective effect against oxidative stress in schizophrenic patients.

\section{Conclusion}

Atypical antipsychotics especially clozapine, olanzapine and quetiapine demonstrate the effective outcome of antipsychotic treatment and beneficial antioxidative action by reducing lipid peroxidation and increasing TAC measured by FRAP.

\section{Acknowledgments}

This work was supported by the National Science Centre, Poland (Grant No. 2011/01/B/NZ4/04903) and Medical University of Lodz, Poland (Grant No.: 503-01-001-1900, 503-01-002/003/004-18).

\section{Author Contributions}

All authors contributed to data analysis, drafting or revising the article, agreed on the journal to which the article will be submitted, gave final approval of the version to be published, and agree to be accountable for all aspects of the work. The authors are grateful to Prof. D. Nowak for his consultations.

\section{Disclosure}

The authors declare no conflicts of interest in this manuscript.

\section{References}

1. Carpenter WT. The deficit syndrome. Am J Psychiatry. 1994;151:327-329.

2. Tamminga CA, Holcomb HH. Phenotype of schizophrenia: a review and formulation. Mol Psychiatry. 2005;10(1):27-39. doi:10.1038/sj. mp.4001563

3. Lieberman JA. Is schizophrenia a neurodegenerative disorder? A clinical and neurobiological perspective. Biol Psychiatry. 1999;46 (6):729-739. doi:10.1016/s0006-3223(99)00147-x

4. Jaaro-Peled H, Sawa A. Neurodevelopmental factors in schizophrenia. Psychiatr Clin N Am. 2020;43:263-274. doi:10.1016/j. psc.2020.02.010.399

5. Gupta S, Kulhara P. What is schizophrenia: a neurodevelopmental or neurodegenerative disorder or a combination of both? A critical analysis. Indian J Psychiatry. 2010;52(1):21-27. doi:10.4103/00195545.58891

6. Davis J, Moylan S, Harvey BH, et al. Neuroprogression in schizophrenia: pathways underpinning clinical staging and therapeutic corollaries. Aust N Z J Psychiatry. 2014;48(6):512-529. doi:10.1177/ 0004867414533012

7. Mayer AR, Ruhl D, Merideth F, et al. Functional imaging of the hemodynamic sensory gating response in schizophrenia. Hum Brain Mapp. 2013;34(9):2302-2312. doi:10.1002/hbm.22065

8. Noto C, Ota VK, Gadelha A, et al. Oxidative stress in drug naïve first episode psychosis and antioxidant effects of risperidone. $J$ Psychiatr Res. 2005;68:210-216. doi:10.1016/j.jpsychires.2015.07.003

9. Dietrich-Muszalska A. Oxidative Stress in Schizophrenia. In: DietrichMuszalska A, Chauhan V, Grignon S, editors. Studies on Psychiatric Disorders. Oxidative Stress in Applied Basic Research and Clinical Practice. New York, Heidelberg, Dordrecht, London: Humana Press, Springer Science+Business Media; 2015:43-72. 
10. Lieberman JA, Bymaster FP, Meltzer HY, et al. Antipsychotic drugs: comparison in animal models of efficacy, neurotransmitter regulation, and neuroprotection. Pharmacol Rev. 2008;60(3):358-403. doi:10.1124/pr.107.00107

11. Carpenter WT, Koenig JI. The evolution of drug development in schizophrenia: past issues and future opportunities. Neuropsychopharmacology. 2008;33(9):2061-2079. doi:10.1038/sj. npp.1301639

12. Kapur S, Mamo D. Half a century of antipsychotics and still a central role for dopamine D2 receptors. Prog Neuropsychopharmacol Biol Psychiatry. 2003;27(7):1081-1090. doi:10.1016/j.pnpbp.2003.09.004

13. Meltzer HY, Massey BW. The role of serotonin receptors in the action of atypical antipsychotic drugs. Curr Opin Pharmacol. 2011;11(1):59-67. doi:10.1016/j.coph.2011.02.007

14. Farah A. Atypicality of atypical antipsychotics. Prim Care Companion J Clin Psychiatry. 2005;7(6):268-274. doi:10.4088/pcc.v07n0602

15. Gardner I, Zahid N, MacCrimmon D, et al. A comparison of the oxidation of clozapine and olanzapine to reactive metabolites and the toxicity of these metabolites to human leukocytes. Mol Pharmacol. 1998;53(6):991-998.

16. Pillai A, Parikh V, Terry AV Jr, et al. Long-term antipsychotic treatments and crossover studies in rats: differentiation effects of typical agents on the expression of antioxidant enzymes and membrane lipid peroxidation in rat brain. J Psychiatr Res. 2007;41:372-386. doi:10.1016/j.jpsychires.2006.01.011

17. Dietrich-Muszalska A, Olas B, Głowacki R, et al. Oxidative/nitrative modifications of plasma proteins and thiols from patients with schizophrenia. Neuropsychobiology. 2009;59(1):1-7. doi:10.1159/ 000202822

18. Zhang XY, Tan YL, Cao LY. Antioxidant enzymes and lipid peroxidation in different forms of schizophrenia treated with typical and atypical antipsychotics. Schizophr Res. 2006;81(2-3):291-300. doi:10.1016/j.schres.2005.10.011

19. Grignon S, Chianetta JM. Assessment of malondialdehyde levels in schizophrenia: a meta-analysis and some methodological considerations. Prog Neuropsychopharmacol Biol Psychiatry. 2007;31(2):365-369. doi:10.1016/j.pnpbp.2006.09.012

20. Tsai MC, Liou CW, Lin TK, et al. Changes in oxidative stress markers in patients with schizophrenia: the effect of antipsychotic drugs. Psychiatry Res. 2013;209(3):284-290. doi:10.1016/j. psychres.2013.01.023

21. American Psychiatric Association. The Diagnostic and Statistical Manual of Mental Disorders, Fourth Edition (DSM-IV). Washington, DC; 1994:873

22. Simpson GM, Angus JW. A rating scale for extrapyramidal side effects. Acta Psychiatr Scand Suppl. 1970;212:11-19. doi:10.1111/ j.1600-0447.1970.tb02066.x

23. Barnes TR. A rating scale for drug-induced akathisia. $\mathrm{Br}$ J Psychiatry. 1989;154:672-676. doi:10.1192/bjp.154.5.672

24. Kay SR, Fiszbein A, Opler LA. The positive and negative syndrome scale (PANNS) for schizophrenia. Schizophr Bull. 1987;13:261-276. doi:10.1093/schbul/13.2.261

25. Sheehan DV, Lecrubier Y, Sheehan KH, et al. The Mini-International Neuropsychiatric Interview (M.I.N.I.): the development and validation of a structured diagnostic psychiatric interview for DSM-IV and ICD-10. J Clin Psychiatry. 1998;59:22-33.

26. Mauri MC, Volonteri LC, Colasanti A, et al. Clinical pharmacokinetics of atypical antipsychotics: a critical review of the relationship between plasma concentrations and clinical response. Clin Pharmacokinet. 2007;46(5):369-388. doi:10.2165/00003088200746050-00001

27. Leucht S, Leucht C, Huhn M, et al. Sixty years of placebo-controlled antipsychotic drug trials in acute schizophrenia: systematic review, bayesian meta-analysis, and meta-regression of efficacy predictors. Am J Psychiatry. 2017;174(10):927-942. doi:10.1176/appi. ajp.2017.16121358
28. Roberts LJ, Morrow JD. Measurement of F(2)-isoprostanes as an index of oxidative stress in vivo. Free Radic. Biol Med. 2000;28 (4):505-513. doi:10.1016/s0891-5849(99)00264-6

29. Montuschi P, Peter J, Barnes PJ, Roberts JII. Isoprostanes: markers and mediators of oxidative stress. FASEB J. 2004;18(15):1791-1800. doi:10.1096/fj.04-2330rev

30. Rice-Evans CA. Formation of free radicals and mechanisms of action in normal biochemical processes and pathological states. In: RiceEvans CA, Burdon RH, editors. Free Radical Damage and Its Control. Amsterdam: Elsevier; 1994:131-153.

31. Wachowicz B, Kustron J. Effect of cisplatin on lipid peroxidation in pig blond platelets. Cytobios. 1992;70(280):41-47.

32. Benzie IF, Strain JJ. The ferric reducing ability of plasma (FRAP) as a measure of "antioxidant power": the FRAP assay. Anal Biochem. 1996;239(1):70-76. doi:10.1006/abio.1996.0292

33. Kolodziejczyk-Czepas J, Nowak P, Kowalska I, et al. Biological activity of clovers-free radical scavenging ability and antioxidant action of six Trifolium species. Pharm Biol. 2014;52 (10):1308-1314. doi:10.3109/13880209.2014.891042

34. Divac N, Prostran M, Jakovcevski I, et al. Second-generation antipsychotics and extrapyramidal adverse effects. Biomed Res Int. 2014:656370. doi: 10.1155/2014/656370.

35. Schatzberg AF, Nemeroff CB. The American Psychiatric Publishing Textbook of Psychopharmacology. Ed. 3. (red.). Arlington: American Psychiatric Publishing, Inc; 2004.

36. Lepping P, Delieu J, Mellor R, Williams JHH, Hudson PR, HunterLavin C. Antipsychotic medication and oxidative cell stress: a systematic review. J Clin Psychiatry. 2011;72(3):273-285. doi:10.4088/JCP.09r05268yel

37. Walss-Bass C, Weintraub ST, Hatch J, et al. Clozapine causes oxidation of proteins involved in energy metabolism: a possible mechanism for antipsychotic-induced metabolic alterations. Int. J Neuropsychopharmacol. 2008;11(8):1097-1104. doi:10.1017/ S1461145708008882

38. Bošković M, Vovk T, Kores Plesničar B, Grabnar I. Oxidative stress in schizophrenia. Curr Neuropharmacol. 2011;9(2):301-312. doi:10.2174/157015911795596595

39. Dietrich-Muszalska A, Olas B. Isoprostanes as indicators of oxidative stress in schizophrenia. World J Biol Psychiatry. 2009;10(1):27-33. doi:10.1080/15622970701361263

40. Dietrich-Muszalska A, Kontek B. Lipid peroxidation in patients with schizophrenia. Psychiatry Clin Neurosci. 2010;64(5):469-475. doi:10.1111/j.1440-1819.2010.02132.x

41. Huang TL, Liou C, Lin TK. Serum thiobarbituric acid-reactive substances and free thiol levels in schizophrenia patients: effects of antipsychotic drugs. Psychiatry Res. 2010;177(1-2):18-21. doi:10.1016/j.psychres.2009.01.017

42. Zhang XY, Tan YL, Cao LY, et al. Antioxidant enzymes and lipid peroxidation in different forms of schizophrenia treated with typical and atypical antipsychotics. Schizophr Res. 2006;81(2-3):291-300. doi:10.1016/j.schres.2005.10.011

43. Hendouei N, Farnia S, Mohseni F, et al. Alterations in oxidative stress markers and its correlation with clinical findings in schizophrenic patients consuming perphenazine, clozapine and risperidone. Biomed Pharmacother. 2018;103:965-972. doi:10.1016/j. biopha.2018.04.109

44. Medina-Hernández V, Ramos-Loyo J, Luquin S, et al. Increased lipid peroxidation and neuron specific enolase in treatment refractory schizophrenics. J Psychiatr Res. 2007;41(8):652-658. doi:10.1016/j. jpsychires.2006.02.010

45. Dietrich-Muszalska A, Bartosz G, Sadowska-Bartosz I. The role of nitric oxide and nitrosative stress in schizophrenia. In: DietrichMuszalska A, Chauhan V, Grignon S, editors. Studies on Psychiatric Disorders. New York, Heidelberg, Dordrecht, London: Humana Press, Springer; 2015:299-326. 
46. Dietrich-Muszalska A, Olas B, Rabe-Jablonska J. Oxidative stress in blood platelets from schizophrenic patients. Platelets. 2005;16 (7):386-391. doi:10.1080/09537100500128872

47. Kim HK, Andreazza AC, Yeung PY, et al. Oxidation and nitration in dopaminergic areas of the prefrontal cortex from patients with bipolar disorder and schizophrenia. $J$ Psychiatry Neurosci. 2014;39 (4):276-285. doi:10.1503/jpn.130155

48. Ustundag B, Atmaca M, Kirtas O, et al. Total antioxidant response in patients with schizophrenia. Psychiatry Clin Neurosci. 2006;60 (4):458-464. doi:10.1111/j.1440-1819.2006.01532.x

49. Gilca M, Piriu G, Gaman, et al. A study of antioxidant activity in patients with schizophrenia taking atypical antipsychotics. Psychopharmacology. 2014;231:4703-4710. doi:10.1007/s00213014-3624-0
50. Sadowska-Bartosz I, Galiniak S, Bartosz G, et al. Antioxidant properties of atypical antipsychotic drugs used in the treatment of schizophrenia. Schizophr Res. 2016;176(2-3):245-251. doi:10.1016/ j.schres.2016.07.010

51. Dietrich-Muszalska A, Kolinska-Łukaszuk J. Comparative effects of aripiprazole and selected antipsychotic drugs on lipid peroxidation in plasma. Psychiatry Clin Neurosci. 2017. doi:10.1111/pcn.1263

52. Virit O, Altindag A, Yumru M, et al. A defect in the antioxidant defense system in schizophrenia. Neuropsychobiology. 2009;60 (2):87-93. doi:10.1159/000239684

53. Chalabi BM AL, Thanoon IAJ, Ahmed FA. Potential effect of olanzapine on total antioxidant status and lipid peroxidation in schizophrenic patients. Neuropsychobiology. 2009;59(1):8-11. doi:10.1159/ 000202823

\section{Publish your work in this journal}

Neuropsychiatric Disease and Treatment is an international, peerreviewed journal of clinical therapeutics and pharmacology focusing on concise rapid reporting of clinical or pre-clinical studies on a range of neuropsychiatric and neurological disorders. This journal is indexed on PubMed Central, the 'PsycINFO' database and CAS, and is the official journal of The International Neuropsychiatric Association (INA). The manuscript management system is completely online and includes a very quick and fair peer-review system, which is all easy to use. Visit http://www.dovepress.com/testimonials.php to read real quotes from published authors. 